\title{
Higher-Order Iterative Learning Control with Optimal Control Gains Based on Evolutionary Algorithm for Nonlinear System
}

\author{
Yun-Shan Wei $\mathbb{D},{ }^{1}$ Xiaofen Yang, ${ }^{2}$ Wenli Shang $\mathbb{D},{ }^{1}$ and Ying-Yu Chen ${ }^{1}$ \\ ${ }^{1}$ School of Electronics and Communication Engineering, Guangzhou University, Guangzhou 510006, China \\ ${ }^{2}$ School of Mechanical and Electric Engineering, Guangzhou University, Guangzhou 510006, China \\ Correspondence should be addressed to Wenli Shang; shangwl@gzhu.edu.cn
}

Received 12 October 2021; Accepted 20 December 2021; Published 30 December 2021

Academic Editor: Zhenyu Lu

Copyright (C) 2021 Yun-Shan Wei et al. This is an open access article distributed under the Creative Commons Attribution License, which permits unrestricted use, distribution, and reproduction in any medium, provided the original work is properly cited.

For the nonlinear discrete-time system, higher-order iterative learning control (HOILC) with optimal control gains based on evolutionary algorithm (EA) is developed in this paper. Since the updating actions are constituted by the tracking information from several previous iterations, the suitably designed HOILC schemes with appropriate control gains usually achieve fast convergence speed. To optimize the control gains in HOILC approach, EA is introduced. The encoding strategy, population initialization, and fitness function in EA are designed according to the HOILC characteristics. With the global optimization of EA, the optimal control gains of HOILC are selected adaptively so that the number of convergence iteration is reduced in ILC process. It is shown in simulation that the sum absolute error, total square error, and maximum absolute error of tracking in the proposed HOILC based on EA are convergent faster than those in conventional HOILC.

\section{Introduction}

In real applications such as robot manipulator systems [1-5] and flexible systems [6-8], there are many unmanned autonomous systems in complex environments. The exact mathematical model is hard to construct. For these systems, iterative learning control (ILC) is proposed. It is an effective intelligent control approach applied in dynamical systems that perform repetitive tasks to track a specific trajectory in a certain time interval. By using the control input and tracking information of previous iterations, the control input signal can be gradually updated from iteration to iteration such that the tracking performance can be improved. Less previous knowledge about the controlled systems makes ILC popular in theoretical fields [9-14] as well as applicable fields [15-19].

First-order ILC, which generates the control input from tracking information at last iteration, is widely applied to dynamical systems for perfect tracking in a finite time interval [20-26]. However, only the tracking information of last iteration is utilized to update the current control input in first-order ILC, and thus it is difficult to obtain a satisfactory convergence speed. To achieve faster convergence speed, higher-order ILC (HOILC) adopting the tracking information of many previous iterations to generate the current control input signal was proposed [27-31]. Since the updating actions are constituted by the tracking information from several previous iterations, the tracking performance of suitably designed HOILC is better than that of first-order ILC. Specifically, the appropriate control gains can accelerate the convergence process of HOILC. Thus, how to select optimal control gains is a significant issue in HOILC designs.

Motivated by the above observation, in this paper, the evolutionary algorithm (EA) originating from biological evolutionism is adopted to choose the optimal control gains in HOILC scheme adaptively. EA is a heuristic optimizing algorithm which simulates the reproduction, selection, crossover, and mutation in biological evolution process. It has been widely introduced to deal with various optimal issues [32-34]. In this paper, the encoding strategy, population initialization, and fitness function of EA are designed according to the HOILC characteristics such that the generations in EA are reduced. Then, the designed EA is involved in HOILC to optimize the control gains. After that, 
the optimal control gains and the control inputs are generated simultaneously. Comparing with traditional HOILC, the number of convergence iteration is reduced in the proposed EA scheme based HOILC (EA-HOILC). The EA with global optimization is introduced to optimize the control gains of HOILC in this paper.

The rest of paper is organized as follows. The problem formulation is given in Section 2. The HOILC with its convergence analysis is provided in Section 3. Section 4 presents the designed EA-HOILC scheme with optimal control gains. In Section 5, an example is provided to illustrate the effectiveness of the proposed EA-HOILC. Section 6 concludes this paper.

\section{Problem Formulation}

Consider the following nonlinear discrete-time system which performs repetitive operation:

$$
\left\{\begin{array}{l}
x_{j}(i+1)=f\left(x_{j}(i), i\right)+B(i) u_{j}(i) \\
y_{j}(i)=C(i) x_{j}(i)
\end{array}\right.
$$

where $j \in\{0,1,2, \ldots\}$ and $i \in\{0,1, \ldots, M\}$ represent the iteration index and the time point, respectively. $x_{j}(i) \in R^{w}$, $u_{j}(i) \in R$, and $y_{j}(i) \in R$ denote the state, control input, and output of system (1), respectively. $B(i) \in R^{w}, C(i) \in R^{1 \times w}$, and $f(\cdot, \cdot) \in R^{w} \cdot y_{d}(i)=C(i) x_{d}(i)$ for $i \in\{0,1, \ldots, M\}$ is the reference output, where $x_{d}(i)$ is the corresponding reference state. $e_{j}(i)=y_{d}(i)-y_{j}(i)$ is the ILC tracking error at $j$ th iteration for $i \in\{0,1, \ldots, M\}$. The following assumptions are required for the technical analysis. $\|\cdot\|$ represents the required norm in this paper.

Assumption 1. For all $j$, the initial state satisfies

$$
x_{j}(0)=x_{d}(0) \text {. }
$$

As the identical initial condition considered in Assumption 1 cannot be satisfied, the techniques proposed in [29-31] can be introduced to deal with the vibration of initial state.

Assumption 2. The nonlinear function $f(\cdot, \cdot)$ in system (1) is assumed to be differentiable to $i$ and to be globally Lipschitz in the first variable, that is, $\forall \bar{x}, \bar{x} \in R^{w}$,

$$
\|f(\bar{x}, i)-f(\breve{x}, i)\| \leq c_{f}\|\bar{x}-\bar{x}\|,
$$

where $c_{f}>0$ is the Lipschitz constant.

Assumption 3. The number $C(i+1) B(i) \neq 0$.

Remark 1. It is noted that Assumption 3 implies that the relative degree of system (1) is one. For the nonlinear discrete-time systems with higher relative degree, the ILC law can be modified according to the order of system relative degree as discussed in [31].

Suppose that the reference output $y_{d}(i)$ is realizable, there exists a unique control input $u_{d}(i) \in R$ such that

$$
\left\{\begin{array}{l}
x_{d}(i+1)=f\left(x_{d}(i), i\right)+B(i) u_{d}(i), \\
y_{d}(i)=C(i) x_{d}(i)
\end{array}\right.
$$

The objective of this paper is to develop an EA-HOILC method, which generates the control input from the tracking information of several previous iterations. The control gains are optimized by EA to reduce the number of convergence iteration. For HOILC convergence analysis, the following lemma is adopted.

Lemma 1 (see [31]). Let $\left\{g_{j}\right\}$ be a real sequence defined as

$$
g_{j} \leq \omega_{1} g_{j-1}+\omega_{2} g_{j-2}+\cdots+\omega_{N} g_{j-N}+d_{j},
$$

for $j \geq N+1$, where $d_{j}$ is a specific real sequence. If $\omega_{1}, \omega_{2}, \ldots, \omega_{N}$ are nonnegative numbers satisfying

$$
\omega=\sum_{v=1}^{N} \omega_{v}<1
$$

then $\lim _{j \longrightarrow+\infty} d_{j}=0$ implies that $\lim _{j \longrightarrow+\infty} g_{j}=0$.

\section{HOILC Design and Convergence Analysis}

In this section, for nonlinear discrete-time system (1) under Assumptions 1-3, the following HOILC law is designed for $i \in\{0,1, \ldots, M\}$ and $j \geq N-1$ :

$$
u_{j+1}(i)=\sum_{v=1}^{N} W_{v} u_{j-v+1}(i)+\sum_{v=1}^{N} L_{v} e_{j-v+1}(i+1),
$$

where $N \geq 1$ is the order of HOILC law (7), and $W_{v} \in R$ and $L_{v} \in R\left(\left|L_{v}\right| \leq s_{L}\right)$ for $v=1,2, \ldots, N$ are the control gains.

Remark 2. In the existing HOILC schemes [30, 31], the initial control inputs $u_{0}(i), u_{1}(i), \ldots, u_{N-1}(i)$ are normally set as zero vectors. In this paper, since the control inputs can be obtained by EA along with the optimal control gains, we can set the initial control inputs $u_{0}(i), u_{1}(i), \ldots, u_{N-1}(i)$ same as the generated control inputs. It means that the initial control inputs are optimized by EA, which can also accelerate the convergence speed.

Theorem 1. For nonlinear discrete-time system (1) under Assumptions 1-3, the HOILC law (7) is applied. If the control gains $W_{v}$ and $L_{v}\left(\left|L_{v}\right| \leq s_{L}\right)$ for $v=1,2, \ldots, N$ are selected to make

$$
\begin{gathered}
\sum_{v=1}^{N} W_{v}=1, \\
\left|W_{v}-L_{v} C(i+1) B(i)\right| \leq \gamma_{v}, \\
\sum_{v=1}^{N} \gamma_{v}<1,
\end{gathered}
$$

then $\lim _{j \longrightarrow+\infty} e_{j}(i)=0$ for $i \in\{0,1, \ldots, M+1\}$. 
Proof. Let $\delta u_{j}(i)=u_{d}(i)-u_{j}(i)$ and $\delta x_{j}(i)=x_{d}(i)-x_{j}$

(i). Subtracting both sides of (7) with $u_{d}(i)$ and considering

(1), (4), and (8), we obtain

$$
\begin{aligned}
& \delta u_{j+1}(i)=\sum_{v=1}^{N} W_{v} \delta u_{j-v+1}(i)-\sum_{v=1}^{N} L_{v} e_{j-v+1}(i+1), \\
& =\sum_{v=1}^{N} W_{v} \delta u_{j-v+1}(i)-\sum_{v=1}^{N} L_{v} C(i+1) \delta x_{j-v+1}(i+1), \\
& =\sum_{v=1}^{N} W_{v} \delta u_{j-v+1}(i)-\sum_{v=1}^{N} L_{v} C(i+1)\left[f\left(x_{d}(i), i\right)-f\left(x_{j-v+1}(i), i\right)+B(i) \delta u_{j-v+1}(i)\right], \\
& =\sum_{v=1}^{N}\left[W_{v}-L_{v} C(i+1) B(i)\right] \delta u_{j-v+1}(i)-\sum_{v=1}^{N} L_{v} C(i+1)\left[f\left(x_{d}(i), i\right)-f\left(x_{j-v+1}(i), i\right)\right] .
\end{aligned}
$$

Then, noting convergence condition (9) and Assumption 2 , we can further deduce that

$$
\begin{aligned}
\left|\delta u_{j+1}(i)\right| & \leq \sum_{v=1}^{N} \gamma_{v}\left|\delta u_{j-v+1}(i)\right| \\
& +\sum_{v=1}^{N} s_{L} s_{C} c_{f}\left\|\delta x_{j-v+1}(i)\right\|
\end{aligned}
$$

where $\|C(i)\| \leq s_{C}$ and $\left|L_{v}\right| \leq s_{L}$ for $v=1,2, \ldots, N$.

On the other hand, it follows from (1) and (4) that

$$
\begin{aligned}
\delta x_{j}(i)= & f\left(x_{d}(i-1), i-1\right) \\
& -f\left(x_{j}(i-1), i-1\right)+B(i-1) \delta u_{j}(i-1) .
\end{aligned}
$$

Taking norm on both sides of (13) and considering Assumptions 1-2, it yields

$$
\begin{aligned}
\left\|\delta x_{j}(i)\right\| & \leq c_{f}\left\|\delta x_{j}(i-1)\right\|+s_{B}\left|\delta u_{j}(i-1)\right| \\
& \leq c_{f}^{2}\left\|\delta x_{j}(i-2)\right\|+c_{f} s_{B}\left|\delta u_{j}(i-2)\right|+s_{B}\left|\delta u_{j}(i-1)\right| \\
& \leq \cdots \cdots \\
& \leq c_{f}^{i}\left\|\delta x_{j}(0)\right\|+\sum_{k=0}^{i-1} c_{f}^{i-k-1} s_{B}\left|\delta u_{j}(k)\right| \\
& =\sum_{k=0}^{i-1} c_{f}^{i-k-1} s_{B}\left|\delta u_{j}(k)\right|,
\end{aligned}
$$

where $\|B(i)\| \leq s_{B}$. Substituting (14) into (12),

$$
\begin{aligned}
\left|\delta u_{j+1}(i)\right| & \leq \sum_{v=1}^{N} \gamma_{v}\left|\delta u_{j-v+1}(i)\right| \\
& +\sum_{v=1}^{N} \sum_{k=0}^{i-1} s_{L} s_{C} s_{B} c_{f}^{i-k}\left|\delta u_{j-v+1}(k)\right|
\end{aligned}
$$

As $i=0$, considering (2) of Assumption 1, it is derived from (12) that

$$
\left|\delta u_{j+1}(0)\right| \leq \sum_{v=1}^{N} \gamma_{v}\left|\delta u_{j-v+1}(0)\right| .
$$

Applying Lemma 1 to (16) with convergence condition (10), we have

$$
\lim _{j \longrightarrow+\infty}\left|\delta u_{j}(0)\right|=0
$$

As $i=1$, from (16), there is

$$
\begin{aligned}
\left|\delta u_{j+1}(1)\right| & \leq \sum_{v=1}^{N} \gamma_{v}\left|\delta u_{j-v+1}(1)\right| \\
& +\sum_{v=1}^{N} s_{L} s_{C} s_{B} c_{f}\left|\delta u_{j-v+1}(0)\right| .
\end{aligned}
$$

Applying Lemma 1 to (18) with convergence condition (10) and considering (17), we obtain

$$
\lim _{j \longrightarrow+\infty}\left|\delta u_{j}(1)\right|=0 \text {. }
$$

Assume that for $i=1,2, \cdots, n-1$, there is

$$
\lim _{j \longrightarrow+\infty}\left|\delta u_{j}(i)\right|=0 \text {. }
$$

As $i=n$, it follows from (15) that

$$
\left|\delta u_{j+1}(n)\right| \leq \sum_{v=1}^{N} \gamma_{v}\left|\delta u_{j-v+1}(n)\right|+\sum_{v=1}^{N} \sum_{k=0}^{n-1} s_{L} s_{C} s_{B} c_{f}^{n-k}\left|\delta u_{j-v+1}(k)\right| .
$$

Applying Lemma 1 to (21) with convergence condition (10) and considering (17) and (20), we can derive

$$
\lim _{j \longrightarrow+\infty}\left|\delta u_{j}(n)\right|=0 \text {. }
$$

Finally, based on the mathematical induction, the following result can be deduced:

$$
\lim _{j \longrightarrow+\infty}\left|\delta u_{j}(i)\right|=0, i \in\{0,1, \ldots, M\} .
$$

Noting (2) in Assumption 1, then it can be obtained from (15) and (24) that 


$$
\lim _{j \longrightarrow+\infty}\left\|\delta x_{j}(i)\right\|=0, i \in\{0,1, \ldots, M+1\}
$$

Furthermore, for $i \in\{0,1, \ldots, M+1\}$, it follows from (1) and (4) that

$$
\lim _{j \longrightarrow+\infty}\left|e_{j}(i)\right| \leq \lim _{j \longrightarrow+\infty} s_{C}\left\|\delta x_{j}(i)\right\|=0
$$

Then, we have $\lim _{j \longrightarrow+\infty} e_{j}(i)=0$ for $i \in\{0,1, \ldots, M+1\}$. The proof is completed.

\section{EA-HOILC Scheme with Optimal Control Gains}

Theorem 1 provides the asymptotic convergence of the proposed HOILC. It is well known that the control gains can affect the convergence performance significantly. In this section, the control gains of the HOILC developed in Section 3 are optimized by EA to reduce the number of convergence iteration.

EA is an intelligent optimization algorithm which simulates the process of biological evolution to gain the optimal solution. The main idea of EA-HOILC is presented as follows.

4.1. Encoding Strategy. In this paper, the control gains of HOILC are real numbers, so it is appropriate to choose the real encoding strategy. The control gains to be optimized in HOILC law (7) are $W_{v}$ and $L_{v}(v=1,2, \ldots, N)$. Since the convergence condition (8) holds, it is easily obtained that $W_{N}=1-\sum_{v=1}^{N-1} W_{v}$. As a result, we can assume the variable vector in EA to be $\lambda \in R^{1 \times(2 N-1)}$, and the encoding strategy is represented as

$$
\lambda=\left[\begin{array}{llllllll}
W_{1} & W_{2} & \cdots & W_{N-1} & L_{1} & L_{2} & \cdots & L_{N}
\end{array}\right] .
$$

4.2. Population Initialization and Individual Evaluation. Based on the convergence conditions (8)-(10), the value range of control gains $W_{v}$ and $L_{v}$ for $v=1,2, \ldots, N$ could be determined. Thus, the initial population can be produced according to the convergent conditions. Let $P$ be the population size, without loss of generality, assume that $P$ is even. The variable vector of $h$ th individual in the population is represented as $\lambda_{h}=\left[\begin{array}{llllll}W_{1, h} & W_{2, h} & \cdots & W_{N-1, h} & L_{1, h} & L_{2, h}\end{array}\right.$ $\left.L_{N, h}\right] \in R^{1 \times(2 N-1)}$ which are initialized to $\lambda_{h}^{\text {ini }} \in R^{1 \times(2 N-1)}$ for $h=1,2, \ldots, P$. On the other hand, for variable $\lambda_{h}$, let the system output for $h$ th individual at $i$ th time point be $Y_{h}\left(i, \lambda_{h}\right)$. To evaluate the individual superiority, the following fitness function fit $_{h}\left(\lambda_{h}\right) \in R$ of $h$ th individual is established:

$$
\mathrm{fit}_{h}\left(\lambda_{h}\right)=\bar{M}-E_{h}\left(\lambda_{h}\right)
$$

where $\bar{M}$ is a constant large enough and $E_{h}\left(\lambda_{h}\right)$ is the sum of absolute value of tracking error represented as

$$
E_{h}\left(\lambda_{h}\right)=\sum_{i=0}^{M}\left|y_{d}(i)-Y_{h}\left(i, \lambda_{h}\right)\right|
$$

From the fitness function (27) and the initial variable $\lambda_{h}^{\text {ini }}$, the initial fitness value of $h$ th individual fit ${ }_{h}^{\text {ini }}$ is obtained. Then, we have the following initial fitness vector fit ${ }^{\text {ini }} \in R^{P}$ of population:

$$
\mathrm{fit}^{\mathrm{ini}}=\left[\begin{array}{llll}
\mathrm{fit}_{1}^{\mathrm{ini}} & \mathrm{fit}_{2}^{\mathrm{ini}} & \cdots & \mathrm{fit}_{P}^{\text {ini }}
\end{array}\right]^{T} .
$$

Hence, the initial population $\operatorname{pop}^{\text {ini }} \in R^{P \times 2 N}$ is constructed as

$$
\operatorname{pop}^{\text {ini }}=\left[\lambda^{\text {ini }} \text { fit }^{\text {ini }}\right]
$$

where the initial variable of population is

$$
\lambda^{\text {ini }}=\left[\begin{array}{llll}
\lambda_{1}^{\text {ini }} & \lambda_{2}^{\text {ini }} & \cdots & \lambda_{P}^{\text {ini }}
\end{array}\right]^{T} .
$$

From (31), the $\alpha$ th $(1 \leq \alpha \leq 2 N-1)$ variable of the $\beta$ th $(1 \leq \beta \leq P)$ individual is represented as the $\beta$ th row of $\alpha$ th column. The last column of pop ${ }^{\text {ini }}$ shown in (30) is the fitness value of the corresponding initial variable in population.

4.3. Selection Strategy. The individuals are selected into next generation by roulette strategy and elitism strategy. The individual with bigger fitness value is selected at higher probability by the roulette strategy. However, one shortcoming of the roulette strategy is that the best individual in old population might be missed. So, we adopt elitism strategy to ensure that the best individual of last generation can be retained. Due to these two strategies, the number of convergence generation of EA can be reduced.

4.4. Crossover Operator. The crossover probability $0<p_{c}<1$ depends whether an individual needs to cross. For $h^{\prime}$ th individual, where $h^{\prime}=1,3,5, \ldots, P-1$, a random number is produced between 0 and 1 , which is represented as $r_{h^{\prime}}$. If $r_{h^{\prime}}>p_{c}$, the crossover operation occurs. Otherwise, the crossover operation does not occur. Due to the real encoding strategy, arithmetical crossover operator is adopted. Assume the variable vectors of $h^{\prime}$ th and $h^{\prime}+1$ th parent individuals to be par_ $\lambda_{h^{\prime}}$ and par_ $\lambda_{h^{\prime}+1}$, respectively, which are selected to cross. After crossover, they generate two new individuals, of which the variable vectors are represented as chi $\lambda_{h^{\prime}}$ and chi $\lambda_{h^{\prime}+1}$. For $h^{\prime}=1,3,5, \ldots, P-1$, the crossover operation is expressed as

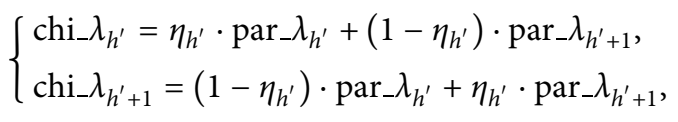

where $\eta_{h^{\prime}} \in(0,1)$ is the crossover weighting for the $h^{\prime}$ th and $h^{\prime}+1$ th parent individuals.

4.5. Mutation Operator. In this paper, we adopt the stochastic mutation strategy. Let $0<p_{m}<1$ be the mutation probability. For $h$ th individual, a number is produced randomly between 0 and 1 represented as $r_{h}, h=1,2, \ldots, P$. If $r_{h}>p_{m}$, the mutation operation occurs. Otherwise, the 
mutation operation does not occur. Let $\operatorname{par} \_\lambda_{h}$ be the variable vector of $h$ th individual which is selected to mutate. After mutation, a new variable vector $c h i_{-} \lambda_{h}$ is produced. The mutation operator is defined as

$$
\text { chi } \_\lambda_{h}=\text { par } \_\lambda_{h}+m_{h}, h=1,2, \ldots, P,
$$

where $m_{h} \in R^{1 \times(2 N-1)}$ is the mutation weighting for $h$ th individual.

4.6. Terminative Conditions. The terminative conditions can be determined by the fitness value or by the tracking error. In simulation, the number of generation in EA is set to be 100 . Finally, we can obtain the optimal control gains $W_{v}^{\text {best }}$ $(v=1,2, \ldots, N-1)$ and $L_{v}^{\text {best }}(v=1,2, \ldots, N)$ from the best individual produced by EA. According to the convergence condition (8), the last control gain $W_{N}^{\text {best }}$ is derived by $W_{N}^{\text {best }}=1-\sum_{v=1}^{N-1} W_{v}^{\text {best }}$.

4.7. Overview of the Proposed EA-HOILC. The flowchart of the proposed EA-HOILC is depicted in Figure 1. First of all, according to the control gains characteristics and convergence conditions (9) and (10), the initial variable $\lambda_{h}^{\text {ini }}$ $(h=1,2, \ldots, P)$ is obtained. Then, we apply the traditional HOILC at $i \in\{0,1, \ldots, M\}$ with initial control gains in each $\lambda_{h}^{\text {ini }}$, where convergence condition (8) is considered. By using the tracking error $E_{h}\left(\lambda_{h}^{\text {ini }}\right)$ with $\lambda_{h}^{\text {ini }}$ as shown in (29) produced by HOILC, the corresponding fitness fit $_{h}$ ini $^{-}$ $(h=1,2, \ldots, P)$ is derived from (27). Combining $\lambda_{h}^{\text {ini }}$ and fit $_{h}^{\text {ini }}$ for $h=1,2, \ldots, P$, the initial population $p o p^{i n i}$ as shown in (31) with (30) and (32) is produced. Secondly, selection, crossover, and mutation are performed by the selection strategy, crossover operator, and mutation operator, respectively. After that, a new input $u_{p}(i)$, $i \in\{0,1, \cdots, M\}$, is obtained along with the optimal control gains. Set the initial control inputs of the EA-HOILC $u_{0}(i)=u_{1}(i)=\cdots=u_{N-1}(i)=u_{p}(i), i=0,1, \ldots, M$. Then, the HOILC with optimal control gains process begins.

\section{Simulation}

To verify the effectiveness of the proposed EA-HOILC, a two-link robotic fish is employed. The system dynamic of the two-link robotic fish is described as follows [22]:

$$
M_{f} \dot{V}_{j}(t)=-\xi V_{j}^{2}(t)+F_{j}(t)
$$

where $M_{f}=0.4 \mathrm{~kg}$ is the mass of robotic fish, $\xi=165.7056$ $\mathrm{kg} / \mathrm{m}$ is the water resistance coefficient, $V_{j}(t)$ denotes the velocity, and $F_{j}(t)$ is the forward thrust produced by the tail motion. Let the velocity $V_{j}\left(i \cdot T_{S}\right)$ and the forward thrust
$F_{j}\left(i \cdot T_{S}\right)$ be the system state $x_{j}(i)$ and the control input $u_{j}(i)$, respectively, where $T_{S}=0.1$ is the sampling time. We can discretize system (34) by using $\dot{V}_{j}\left(i \cdot T_{S}\right) \approx$ $\left(V_{j}\left((i+1) \cdot T_{S}\right)-V_{j}\left(i \cdot T_{S}\right)\right) / T_{S}$. Thus, the discrete-time system is

$$
\left\{\begin{array}{l}
x_{j}(i+1)=x_{j}(i)+A_{f} x_{j}^{2}(i)+B_{f} u_{j}(i), \\
y_{j}(i)=x_{j}(i),
\end{array}\right.
$$

where $A_{f}=-\xi \cdot T_{S} / M_{f}$ and $B_{f}=T_{S} / M_{f}$.

The reference output trajectory is represented as

$y_{d}(i)=0.016[1+\sin (2 \pi i / M-\pi / 2)], i \in\{0,1, \ldots, M+1\}$,

with $M=99$. For the proposed HOILC algorithm, set the order $N=2$. The control gains $W_{1}, L_{1}$, and $L_{2}$ are selected by EA. Another control gain $W_{2}=1-W_{1}$ is obtained from convergence condition (8). The crossover probability $p_{c}=0.7$, and the mutation probability $p_{m}=0.3$. To evaluate the tracking performance, three tracking indexes on sum absolute error $S E_{k}$, total square error $T E_{k}$, and maximum absolute error $M E_{k}$ are defined as follows:

$$
\begin{aligned}
S E_{k} & =\sum_{i=0}^{100}\left|e_{j}(i)\right|, \\
T E_{k} & =\sum_{i=0}^{100}\left[e_{j}(i)\right]^{2}, \\
M E_{k} & =\sup _{i \in\{0,1, \ldots, 100\}}\left|e_{j}(i)\right| .
\end{aligned}
$$

In the simulation, the EA-HOILC is run 10 times, and the optimized control gains are shown in Table 1.

Figure 2 exhibits the system output performance at iterations $j=15, j=22$, and $j=39$ by using EA-HOILC with the average values of optimal control gains in 10 times. To compare the convergence speed between EA-HOILC and conventional HOILC with different parameters, the control gains in HOILC proposed in [30] with 2-order are, respectively, chosen as following two cases. Case 1: $W_{1}=0.7$, $W_{2}=0.3, L_{1}=0.3$, and $L_{2}=0.2$ and Case 2: $W_{1}=0.6$, $W_{2}=0.4, L_{1}=0.2$, and $L_{2}=0.1$. The corresponding sum absolute error $S E_{k}$, total square error $T E_{k}$, and maximum absolute error $M E_{k}$ of tracking are shown in Figure 3. From Figure 3, one can observe that the case with lager control gains in $W_{1}, L_{1}$, and $L_{2}$ can achieve faster convergent speed in conventional HOILC. Moreover, it is clearly revealed that the proposed EA-HOILC can make the convergence iterations less than the conventional HOILC with the same order. 


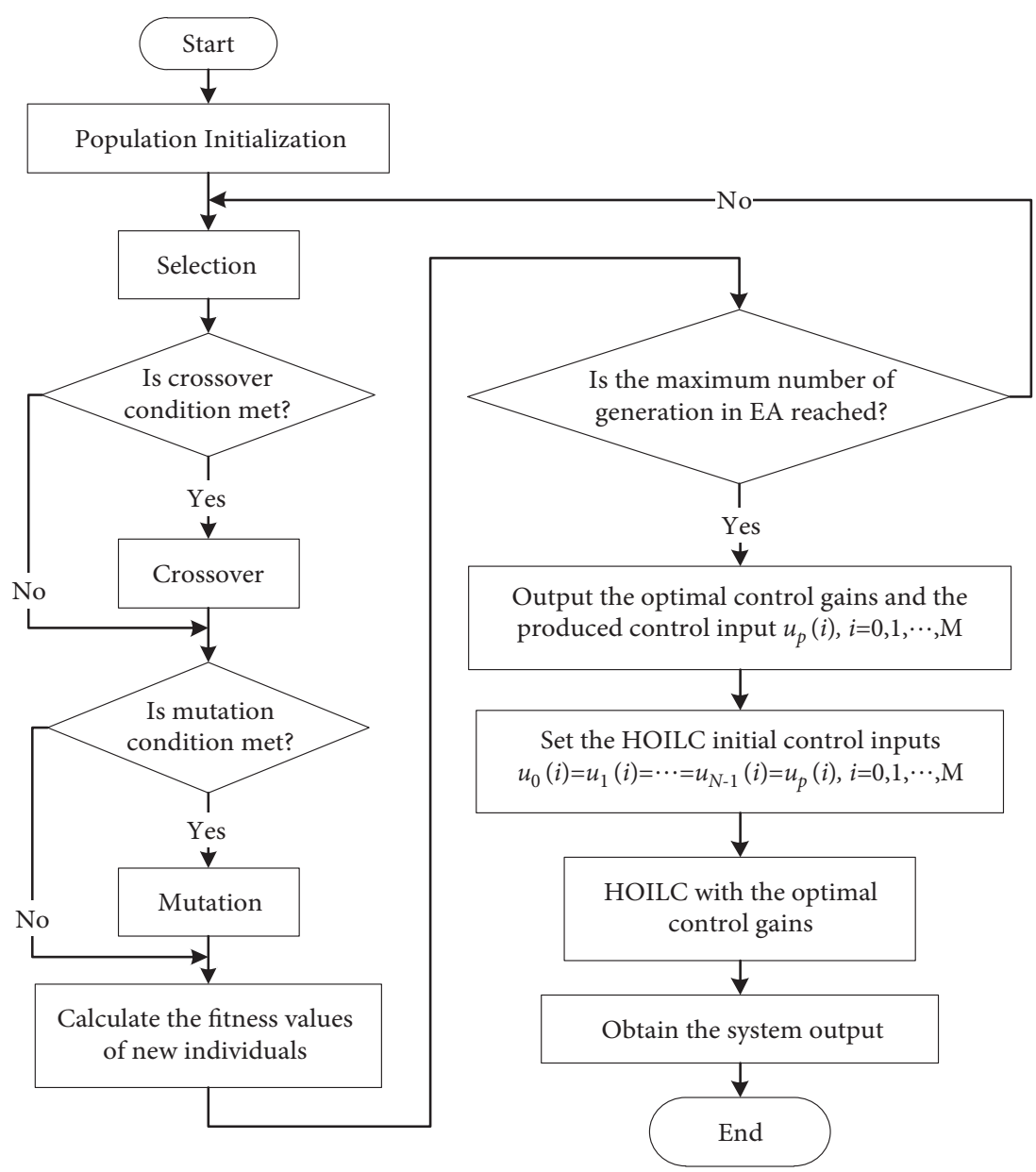

Figure 1: The flowchart of the proposed EA-HOILC.

TABLE 1: Optimized control gains of EA-HOILC in different times.

\begin{tabular}{lcccc}
\hline Times & $W_{1}$ & $W_{2}$ & $L_{1}$ & $L_{2}$ \\
\hline 1 & 0.7474 & 0.2526 & 0.4932 & 0.4570 \\
2 & 0.7827 & 0.2173 & 0.4724 & 0.4924 \\
3 & 0.7313 & 0.2687 & 0.4067 & 0.4068 \\
4 & 0.7972 & 0.2028 & 0.4209 & 0.4856 \\
5 & 0.7161 & 0.2839 & 0.4456 & 0.4177 \\
6 & 0.7243 & 0.2757 & 0.4424 & 0.4744 \\
7 & 0.7465 & 0.2535 & 0.4209 & 0.4678 \\
8 & 0.7502 & 0.2498 & 0.4954 & 0.4682 \\
9 & 0.7543 & 0.2457 & 0.4932 & 0.4156 \\
10 & 0.7474 & 0.2526 & 0.4724 & 0.4570 \\
Average & 0.7827 & 0.2173 & & 0.4569 \\
\hline
\end{tabular}




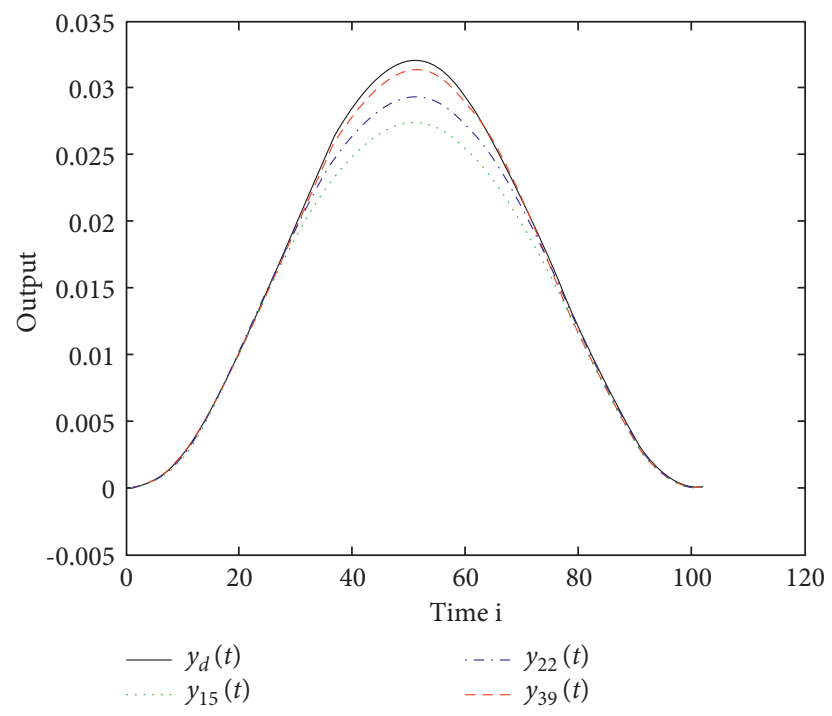

FIgURE 2: Output performance of EA-HOILC with the average values of optimized control gains at iterations $j=15, j=22$, and $j=39$.

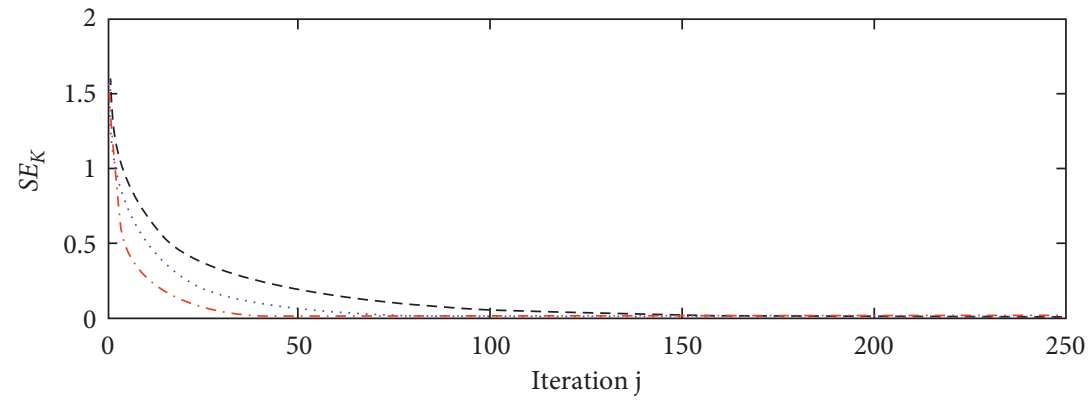

-.. EA-HOILC

..... Conventional HOILC with Case 1

- - - Conventional HOILC with Case 2

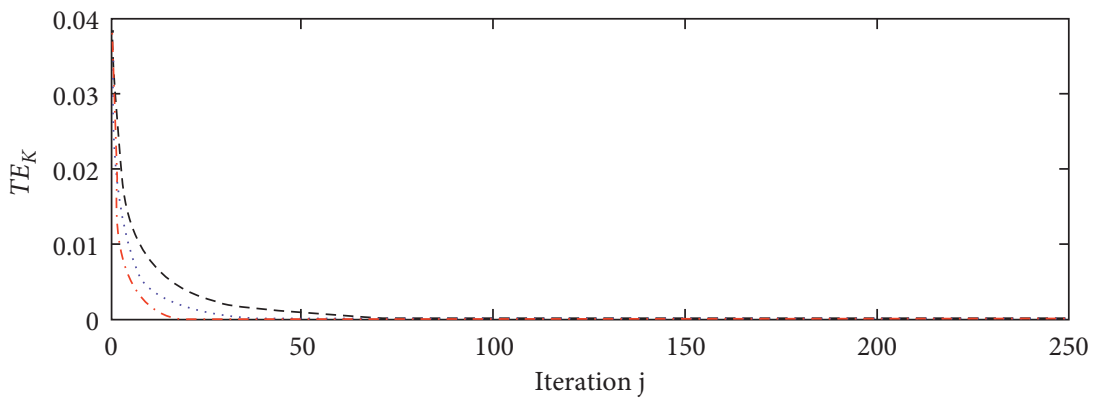

-..- EA-HOILC

..... Conventional HOILC with Case 1

- - - Conventional HOILC with Case 2

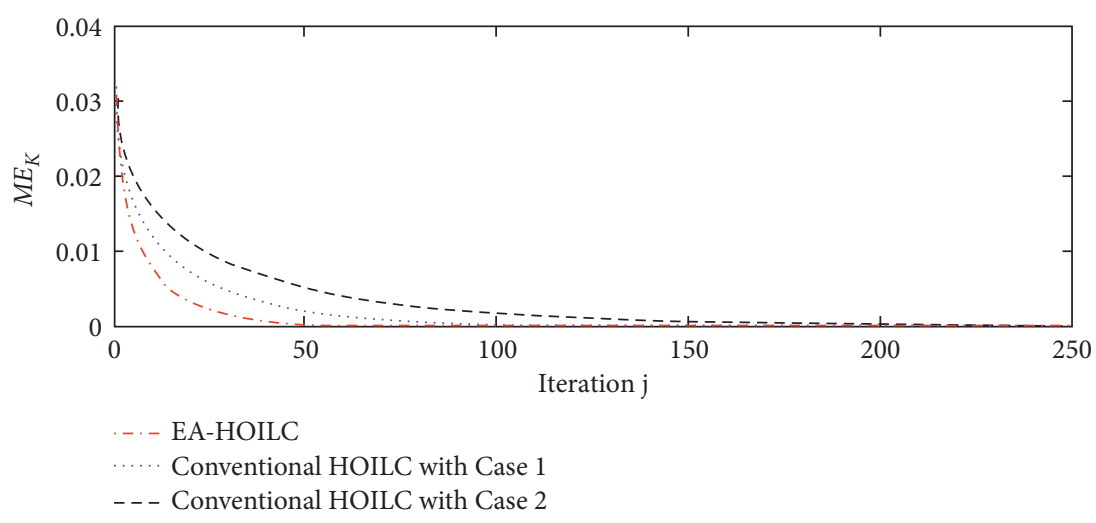

FIGURE 3: The tracking indexes (a) $S E_{k}$, (b) $T E_{k}$, and (c) $M E_{k}$ at different iterations by using EA-HOILC and conventional HOILC with two cases. 


\section{Conclusions}

In this paper, an HOILC law utilizing the tracking information of several previous iterations is proposed for the nonlinear discrete-time system. The convergence is rigorously analyzed based on the mathematical induction. In order to improve the convergence performance of the developed HOILC, the EA with global optimization is introduced to optimize the control gains. With the optimal control gains, the proposed EA-HOILC can achieve faster convergence speed. In simulation, it is shown that the sum absolute error, total square error, and maximum absolute error of tracking in EA-HOILC are convergent faster than those in the conventional HOILC with same order. However, it is worth noting that because EA is adopted to select the control gains, the offline computing time of EA-HOILC is longer. It is very suitable for the cases in which fewer iterations are required only. For instance, to destroy a target with bombs, the proposed method can reduce the number of bombs at the cost of computing time. Future research will extend the EA-HOILC developed in this paper to the dynamical systems with uncertainties in real application [35-37].

\section{Data Availability}

The data used to support the findings of this study are included within the article.

\section{Conflicts of Interest}

The authors declare that there are no conflicts of interest.

\section{Acknowledgments}

This research was funded in part by National Natural Science Foundation of China with grant nos. 61903096 and 62173101, Science and Technology Program of Guangzhou with grant no. 201904010475, and Zhijiang Laboratory's Open Project with grant no. 2021KF0AB06.

\section{References}

[1] G. Peng, C. L. P. Chen, and C. Yang, "Neural networks enhanced optimal admittance control of robot-environment interaction using reinforcement learning," IEEE Transactions on Neural Networks and Learning Systems, pp. 1-11, 2021.

[2] D. Huang, H. Zhan, and C. Yang, "Impedance model-based optimal regulation on force and position of bimanual robots to hold an object," Complexity, vol. 2020, Article ID 3561807 , 13 pages, 2020.

[3] H. Huang, C. Yang, and C. L. P. Chen, "Optimal robot-environment interaction under broad fuzzy neural adaptive control," IEEE Transactions on Cybernetics, vol. 51, no. 7, pp. 3824-3835, 2021.

[4] D. Huang, C. Yang, Y. Pan, and L. Cheng, "Composite learning enhanced neural control for robot manipulator with output error constraints," IEEE Transactions on Industrial Informatics, vol. 17, no. 1, pp. 209-218, 2020.

[5] C. Yang, D. Huang, W. He, and L. Cheng, "Neural control of robot manipulators with trajectory tracking constraints and input saturation," IEEE Transactions on Neural Networks and Learning Systems, vol. 32, no. 9, pp. 4231-4242, 2021.

[6] Z. Zhao, Z. Liu, W. He, K. S. Hong, and H. X. Li, "Boundary adaptive fault-tolerant control for a flexible Timoshenko arm with backlash-like hysteresis," Automatica, vol. 13, Article ID 109690, 2021.

[7] Z. Zhao, C. K. Ahn, and H.-X. Li, "Boundary antidisturbance control of a spatially nonlinear flexible string system," IEEE Transactions on Industrial Electronics, vol. 67, no. 6, pp. 4846-4856, 2020.

[8] Z. Zhao, X. He, and C. K. Ahn, "Boundary disturbance observer-based control of a vibrating single-link flexible manipulator," IEEE Transactions on Systems, Man, and Cybernetics: Systems, vol. 51, no. 4, pp. 2382-2390, 2021.

[9] W. He, T. Meng, S. Zhang, J.-K. Liu, G. Li, and C. Sun, "Dualloop adaptive iterative learning control for a Timoshenko beam with output constraint and input backlash," IEEE Transactions on Systems, Man, and Cybernetics: Systems, vol. 49, no. 5, pp. 1027-1038, 2019.

[10] K. Wan and X.-D. Li, "Robust iterative learning control of 2-D linear discrete FMMII systems subject to iteration-dependent uncertainties," IEEE Transactions on Systems, Man, and Cybernetics: Systems, vol. 51, no. 10, pp. 5949-5961, 2021.

[11] D. Meng and J. Zhang, "Convergence analysis of robust iterative learning control against nonrepetitive uncertainties: system equivalence transformation," IEEE Transactions on Neural Networks and Learning Systems, vol. 32, no. 9, pp. 3867-3879, 2021.

[12] J. Liu, X. Ruan, and Y. Zheng, "Iterative learning control for discrete-time systems with full learnability," IEEE Transactions on Neural Networks and Learning Systems, pp. 1-15, 2020.

[13] J. Chen, C. Hua, and X. Guan, "Iterative learning model-free control for networked systems with dual-direction data dropouts and actuator faults," IEEE Transactions on Neural Networks and Learning Systems, vol. 32, no. 11, pp. 5232-5240, 2021.

[14] Q. Yu, Z. Hou, X. Bu, and Q. Yu, "RBFNN-based data-driven predictive iterative learning control for nonaffine nonlinear systems," IEEE Transactions on Neural Networks and Learning Systems, vol. 31, no. 4, pp. 1170-1182, 2020.

[15] X. Bu, J. Liang, Z. Hou, and R. Chi, "Data-driven terminal iterative learning consensus for nonlinear multiagent systems with output saturation," IEEE Transactions on Neural Networks and Learning Systems, vol. 32, no. 5, pp. 1963-1973, 2021.

[16] X. Jin, "Fault-tolerant iterative learning control for mobile robots non-repetitive trajectory tracking with output constraints," Automatica, vol. 94, pp. 63-71, 2018.

[17] T. Meng, W. He, and X. He, "Tracking control of a flexible string system based on iterative learning control," IEEE Transactions on Control Systems Technology, vol. 29, no. 1, pp. 436-443, 2021.

[18] W. He, T. Meng, X. He, and C. Sun, "Iterative learning control for a flapping wing micro aerial vehicle under distributed disturbances," IEEE Transactions on Cybernetics, vol. 49, no. 4, pp. 1524-1535, 2019.

[19] X. Bu, Q. Yu, Z. Hou, and W. Qian, "Model free adaptive iterative learning consensus tracking control for a class of nonlinear multiagent systems," IEEE Transactions on Systems, Man, and Cybernetics: Systems, vol. 49, no. 4, pp. 677-686, 2019.

[20] X. Li, D. Shen, and B. Ding, "Iterative learning control for output tracking of nonlinear systems with unavailable state 
information," IEEE Transactions on Neural Networks and Learning Systems, pp. 1-8, 2021.

[21] L. Wang, J. Yu, R. Zhang, P. Li, and F. Gao, "Iterative learning control for multiphase batch processes with asynchronous switching," IEEE Transactions on Systems, Man, and Cybernetics: Systems, vol. 51, no. 4, pp. 2536-2549, 2021.

[22] X. Li, Q. Ren, and J. Xu, "Precise speed tracking control of a robotic fish via iterative learning control," IEEE Transactions on Industrial Electronics, vol. 63, no. 4, pp. 2221-2228, 2016.

[23] D. Meng and K. L. Moore, "Contraction mapping-based robust convergence of iterative learning control with uncertain, locally Lipschitz nonlinearity," IEEE Transactions on Systems, Man, and Cybernetics: Systems, vol. 50, no. 2, pp. 442-454, 2020.

[24] X. Wang and J. Wang, "Iterative learning control for onesided Lipschitz nonlinear singular conformable differential equations," International Journal of Robust and Nonlinear Control, vol. 30, no. 17, pp. 7791-7805, 2020.

[25] R. Chi, Y. Lv, and Z. Hou, "Compensation-based data-driven ILC with input and output package dropouts," International Journal of Robust and Nonlinear Control, vol. 30, no. 3, pp. 950-965, 2020.

[26] J. Zhang and D. Meng, "Convergence analysis of saturated iterative learning control systems with locally Lipschitz nonlinearities," IEEE Transactions on Neural Networks and Learning Systems, vol. 31, no. 10, pp. 4025-4035, 2020.

[27] J. Shi, J. Xu, J. Sun, and Y. Yang, "Iterative learning control for time-varying systems subject to variable pass lengths: application to robot manipulators," IEEE Transactions on Industrial Electronics, vol. 67, no. 10, pp. 8629-8637, 2020.

[28] Q. Ai, D. Ke, J. Zuo et al., "High-order model-free adaptive iterative learning control of pneumatic artificial muscle with enhanced convergence," IEEE Transactions on Industrial Electronics, vol. 67, no. 11, pp. 9548-9559, 2020.

[29] D. Meng and J. Zhang, "Robust tracking of nonrepetitive learning control systems with iteration-dependent references," IEEE Transactions on Systems, Man, and Cybernetics: Systems, vol. 51, no. 2, pp. 842-852, 2021.

[30] Y. S. Wei and X. D. Li, "Robust higher-order ILC for nonlinear discrete-time systems with varying trail lengths and random initial state shifts," IET Control Theory \& Applications, vol. 11, no. 15, pp. 2240-2247, 2017.

[31] M. Sun and D. Wang, "Analysis of nonlinear discrete-time systems with higher-order iterative learning control," $D y$ namics and Control, vol. 11, pp. 81-96, 2001.

[32] Z. Song, H. Wang, C. He, and Y. Jin, “A Kriging-assisted two-archive evolutionary algorithm for expensive manyobjective optimization," IEEE Transactions on Evolutionary Computation, vol. 25, no. 6, pp. 1013-1027, 2021.

[33] Y. Tian, X. Zhang, C. Wang, and Y. Jin, "An evolutionary algorithm for large-scale sparse multiobjective optimization problems," IEEE Transactions on Evolutionary Computation, vol. 24, no. 2, pp. 380-393, 2020.

[34] L. Chen, H.-L. Liu, K. C. Tan, and K. Li, "Transfer learning based parallel evolutionary algorithm framework for bi-level optimization," IEEE Transactions on Evolutionary Computation, p. 1, 2021

[35] Z. Zhao and Z. Liu, "Finite-time convergence disturbance rejection control for a flexible Timoshenko manipulator," IEEE/CAA Journal of Automatica Sinica, vol. 8, no. 1, pp. 157-168, 2021.

[36] K. Wan and X. D. Li, "Robust iterative learning control of 2-D linear discrete FMMII systems subject to iteration-dependent uncertainties," IEEE Trans. Syst., Man, Cybern., Syst.vol. 51, no. 3, pp. 1462-1472, 2021.

[37] Z. Zhao, C. K. Ahn, and H.-X. Li, "Dead zone compensation and adaptive vibration control of uncertain spatial flexible riser systems," IEEE, vol. 25, no. 3, pp. 1398-1408, 2020. 